\title{
PANCREATITE AGUDA EM CRIANÇAS: ETIOLOGIA, DIAGNÓSTICO E TRATAMENTO
}

\section{ARTIGO DE REVISÃO}

SPAZIANI, Amanda Oliva ${ }^{1}$

FROTA, Raissa Silva ${ }^{2}$

COGNETTI, Gustavo Barreira ${ }^{3}$

REIS, Gabriela Cardozo dos ${ }^{4}$

CUNHA, Thaís Bortolucci ${ }^{5}$

PEDRÃO, Aline Fróes ${ }^{6}$

SANTOS, Flávio Henrique Nuevo Benez Dos ${ }^{7}$

SPAZIANI, Amanda Oliva. Et al. Pancreatite aguda em crianças: Etiologia, diagnóstico e tratamento. Revista Científica Multidisciplinar Núcleo do Conhecimento. Ano 04, Ed. 09, Vol. 03, pp. 159-167. Setembro de 2019. ISSN: 2448-

\footnotetext{
${ }^{1}$ Graduanda em Medicina pela Universidade Brasil - Fernandópolis, Brasil.

${ }^{2}$ Graduanda em Medicina pela Universidade Brasil - Fernandópolis, Brasil.

${ }^{3}$ Graduando em Medicina pela Universidade Brasil - Fernandópolis, Brasil.

${ }^{4}$ Graduanda em Medicina pela Universidade Brasil - Fernandópolis, Brasil.

${ }^{5}$ Graduanda em Medicina pela Universidade Brasil - Fernandópolis, Brasil.

${ }^{6}$ Graduanda em Medicina pela Universidade Brasil - Fernandópolis, Brasil.

${ }^{7}$ Médico Especialista em Ortopedia e Traumatologia pela Universidade São Francisco - Bragança Paulista, Brasil. Especialista em Medicina Física e Reabilitação pela Rede Lucy Montoro de São Paulo, Brasil. Mestre em Ciências da saúde pela Universidade São Francisco - Bragança Paulista, Brasil.
} 
0959, Link de acesso: https://www.nucleodoconhecimento.com.br/saude/pancreatiteaguda

\section{RESUMO}

Objetivo: O objetivo do presente trabalho é elucidar as causas e manejo clínico de uma criança diagnosticada com pancreatite aguda. Metodologia: Realizou-se um levantamento bibliográfico onde foram selecionados 7 artigos, utilizando-se os descritores: "pancreatite", "pediatria", "diagnóstico de pancreatite" e "tratamento pancreatite aguda" tal como suas traduções na língua inglesa, nos bancos de dados PUBMED, MEDLINE, Lilacs e SciELO, referentes ao período de 2009 e 2019. Conclusão: A etiologia da pancreatite aguda na maioria dos casos não é descoberta a tempo do emprego dos procedimentos para reversão do quadro. No entanto, não apenas é necessário realizar o diagnóstico de forma precoce, como instituir manejo terapêutico e não hesitar em usufruir dos benefícios dos procedimentos cirúrgicos mesmo em casos classificados como mais simples ou com menor gravidade.

Palavras-chave: Pancreatite, pediatria, diagnóstico de pancreatite, tratamento pancreatite aguda.

\section{INTRODUÇÃO}

A Pancreatite Aguda (PA) consiste em uma inflamação do parênquima pancreático, gerando processos fibróticos e inflamatórios, é uma das principais causas de internação devido a doenças do aparelho digestivo, com gasto anual de dois bilhões de dólares nos Estados Unidos. Constata-se aumento na sua incidência, com elevação do número de internações em $20 \%$ nos últimos dez anos ${ }^{1,2}$.

De acordo com o Datasus, no Brasil, no período de julho de 2010 a julho de 2011 foi registrado 25.660 internações, com um custo de mais de 17 bilhões por ano e mortalidade de 5,9\%. Grande parte dos pacientes apresenta a forma leve da pancreatite aguda, que é autolimitada e tem bom prognóstico com o tratamento 
conservador. As formas graves correspondem por 20-30\% dos casos e possuem uma morbimortalidade significativa ${ }^{3}$.

Diferente de como ocorre nos adultos, na maioria dos casos, a causa da PA secundária à doença biliar e alcoolismo, na pediatria as etiologias são variadas, abrangendo inúmeras condições sistêmicas, principalmente secundárias a medicamentos ${ }^{4}$.

As principais manifestações clínicas dessa patologia são dor abdominal arrastada e persistente em conjunto com a perda da funcionalidade do pâncreas. Esses pacientes necessitam frequentemente de procedimentos endoscópicos ou cirúrgicos para identificar e tratar as complicações relacionadas a $\mathrm{PA}^{5}$.

Atualmente, o diagnóstico de pancreatite aguda embasa-se em dois de três critérios entre os seguintes: dor abdominal; aumento dos níveis amilase no sangue em pelo menos três vezes o valor de normalidade; achados compatíveis nos exames de imagem. A evolução é dividida em duas fases na pancreatite aguda: uma precoce, onde a gravidade está associada à resposta inflamatória sistêmica e à falência de órgãos, e uma tardia, onde a evolução pode ser a resolução, estabilização ou necrose ${ }^{3}$.

A necrose pancreática ou do tecido peri-pancreático está relacionada a maior gravidade da PA, sendo que para evolução da pancreatite, a infecção é um fator primordial. Aproximadamente $20 \%$ dos casos cursam com necrose pancreática e um terço dos pacientes evolui com infecção. A mortalidade gira em torno de $30 \%$ na necrose pancreática infectada e quando evolui para disfunção de múltiplos órgãos, pode atingir $70 \%$ mesmo com alta tecnologia das unidades de terapia intensiva. Portanto, a necrose pancreática infectada não deve ser considerada uma afecção benigna $^{5}$.

A identificação da PA é essencial para estabelecer um tratamento adequado e de suporte, diminuindo morbimortalidade dos pacientes ${ }^{6}$. As medidas com mais impacto no manejo da PA são a hidratação, analgesia e nutrição' 


\section{OBJETIVO}

O objetivo do presente trabalho é elucidar as causas e manejo clínico de uma criança diagnosticada com pancreatite aguda.

\section{METODOLOGIA}

Realizou-se um levantamento bibliográfico onde foram selecionados 7 artigos, utilizando-se os descritores: "pancreatite", "pediatria", "diagnóstico de pancreatite" e "tratamento pancreatite aguda" tal como suas traduções na língua inglesa, nos bancos de dados PUBMED, MEDLINE, Lilacs e SciELO, referentes ao período de 2009 e 2019.

Como critério de inclusão optou-se por estudos enfatizando o diagnóstico da pancreatite aguda em crianças, estudos que narrassem as particularidades da abordagem cirúrgica, estudos que abordassem os aspectos etiológicos da pancreatite e estudos acerca dos diagnósticos diferenciais.

Para exclusão foram eleitos os seguintes critérios: estudos com delineamento experimental, pois fugiam do foco desta revisão e estudos que não avaliavam os desfechos: mortalidade, função pancreática, sintomas, qualidade de vida, efeitos adversos e exacerbações em pancreatite aguda.

\section{RESULTADOS E DISCUSSÃO}

Com a busca realizada foram encontrados 31887 estudos, dos quais 7 foram desfrutados para a presente revisão de literatura após a aplicação dos critérios de inclusão e exclusão.

Relatórios publicados nos últimos anos documentam um número crescente de crianças diagnosticadas com pancreatite aguda (PA), pancreatite aguda recorrente (PAD), pancreatite crônica (PC), insuficiência pancreática exócrina (EPI) e outras anormalidades do pâncreas. Apesar da presença de patologias compartilhadas, os 
problemas específicos do pâncreas geralmente são independentes e exigem atenção individual ${ }^{7}$.

O crescente índice de suspeição de Pancreatite Aguda (PA) em crianças chamou a atenção nos últimos anos, bem como foi verificado em diversos estudos o aumento na incidência de casos confirmados da doença. Estudos recentes estimam a incidência de pancreatite aguda (PA) em aproximadamente 1/10.000 crianças por ano, uma incidência que se aproxima da dos adultos ${ }^{8}$.

O maior conhecimento do quadro clínico, bem como o aumento da suspeita dos casos de PA e o uso de drogas que podem causar PA como efeito adverso apresenta como resultado uma elevação no número de diagnósticos da doença, além disso, tem ocorrido doenças sistêmicas em crianças que lesionam o pâncreas de maneira secundária ${ }^{1}$.

As principais etiologias de PA em crianças são as doenças biliares, medicamentosa, PA idiopática, doenças sistêmicas e trauma, seguidas por doenças metabólicas, hereditárias e infecciosas ${ }^{4}$.

Doenças como a obesidade, ressecção ileal, hemolítica crônica, fibrose cística e doença hepática crônica são associados à litíase na infância9 .

Quando é de origem calculosa, a grande maioria dos casos são cálculos pigmentados e a menor parte é de colesterol. A pancreatite biliar aguda na infância possui pequena incidência da forma grave quando comparada aos adultos. O quadro traz a dor abdominal, que ocorre mais difusamente e de forma branda e vômitos na maioria dos casos $^{9}$.

O diagnóstico da PA em pacientes pediátricos acontece, na maioria dos casos, por meio da realização de uma história clínica rica e exames laboratoriais e de imagem

O diagnóstico é clínico, por meio dos sintomas que se assemelham ao que ocorre em adultos, associado com exames laboratoriais (enzimas acinares pancreáticas, amilase e lipase), além dos exames de imagem (ultrassonografia e tomografia de abdome) 
que tem papel crucial no diagnóstico. Dor abdominal e irritabilidade são os achados mais comuns da PA em crianças, seguidos por sensibilidade epigástrica, náusea e vômito. Em bebês e crianças pequenas, os sintomas podem ser sutis; portanto, o diagnóstico requer um alto nível de suspeita ${ }^{1,7,10}$.

Não há diretrizes diagnósticas baseadas em evidências para PA em crianças. A definição do INSPPIRE (Grupo de Estudo Internacional da Pancreatite Pediátrica) de PA pediátrica é uma definição de especialista modelada após os critérios de Atlanta em adultos. De acordo com os critérios da INSPPIRE, um diagnóstico de PA requer pelo menos 2 dos seguintes: (1) dor abdominal compatível com PA, (2) valores de amilase e / ou lipase sérica $\geq 3$ vezes os limites superiores do normal, (3) achados de imagem consistentes com PA. INSPIRE ou outros critérios não abordam as fases (precoce ou tardia) da PA em crianças ou tipos (pancreatite edematosa intersticial, pancreatite necrosante, necrose pancreática infectada) ou gravidade da PA (PA leve, moderada ou grave com falência de órgãos multissistêmica) ${ }^{11}$.

Os exames de imagem desempenham um papel vital na avaliação de crianças com distúrbios pancreáticos conhecidos ou suspeitos. Como complemento aos achados clínicos e laboratoriais, a imagem é capaz de diagnosticar e monitorar com precisão uma ampla gama de doenças pancreáticas, bem como avaliar as complicações associadas. As principais modalidades utilizadas na avaliação radiológica incluem UST, TC e RM, cada uma com suas próprias vantagens e desvantagens na população pediátrica ${ }^{7}$.

O tratamento envolve medidas gerais de hidratação, dieta enteral equilibrada, uso moderado de antibioticoterapia e a correção cirúrgica em casos específicos. Não é indicado frequentemente a colecistectomia em pacientes com PA e barro biliar diagnosticado, com exceção dos casos de PA recorrente? ${ }^{7}$.

As crianças com sinais de desidratação na avaliação inicial são recomendado uma hidratação rápida preferencialmente com soro fisiológico $0,9 \%$ no volume de $20 \mathrm{ml} / \mathrm{kg}$ a cada 20-30 minutos até reversão da desidratação nas avaliações posteriores ${ }^{1}$. 
Para controle da dor abdominal é indicado o uso de analgésicos comuns e se necessário o uso de opioides em doses reduzidas. A dose de Tramal recomendado é de $1 \mathrm{mg} / \mathrm{kg}$ dose no máximo de 6/6 horas e com infusão lenta ${ }^{1}$.

Na parte de nutrição é indicada a administração de dieta enteral até 48 horas após o diagnóstico e a nutrição parenteral fica como alternativa quando o paciente apresentar intolerância a nutrição enteral ${ }^{1}$.

\section{CONCLUSÃO}

Nota-se com os estudos revisados que a pancreatite aguda possui notoriedade na faixa etária pediátrica, principalmente em recém-nascidos. As barreiras para se estabelecer um diagnóstico preciso chama a atenção, ainda mais pela rápida evolução da patologia para estados graves com diversas complicações associadas.

A etiologia da pancreatite aguda na maioria dos casos não é descoberta a tempo do emprego dos procedimentos para reversão do quadro. No entanto, não apenas é necessário realizar o diagnóstico de forma precoce, como instituir manejo terapêutico e não hesitar em usufruir dos benefícios dos procedimentos cirúrgicos mesmo em casos classificados como mais simples ou com menor gravidade.

\section{REFERÊNCIAS}

1. Mekitarian, F.E.; Brunow, C.W.; Duarte, S.F. Pancreatite aguda em pediatria: revisão sistemática da literatura. Jornal de Pediatria, v. 88, n. 2, 2012.

2. Rasslan, R. et al . Necrose pancreática com infecção: estado atual do tratamento. Rev. Col. Bras. Cir., Rio de Janeiro , v. 44, n. 5, p. 521529, Oct. 2017.

3. Cunha, E.F.C. et al . Necrose pancreática delimitada e outros conceitos atuais na avaliação radiológica da pancreatite aguda. Radiol Bras, São Paulo, v. 47, n. 3, p. 165-175, June 2014.

4. Lopez, M.J. The changing incidence of acute pancreatitis in children: a singleinstitution perspective. J Pediatr. 2002;140:622-4. 
5. Cazzo, E. et al . Tratamento Das Fístulas Pancreaticopleurais Secundárias À Pancreatite Crônica. Arq. Bras. Cir. Dig., São Paulo , v. 30, n. 3, p. 225228, Sept. 2017.

6. Andersson, R.; Swärd, A.; Tingstedt, B.; Akerberg, D. Treatment of acute pancreatitis: focus on medical care. Drugs. 2009;69:505-14.

7. Lin, T.K.; Troendle, D.M.; Wallihan, D.B.; Barth, B.; Fox, V.L.; Fishman, D.S.; Morinville, V.D. Specialized Imaging and Procedures in Pediatric Pancreatology: A North American Society for Pediatric Gastroenterology, Hepatology, and Nutrition Clinical Report; Journal of Pediatric Gastroenterology and Nutrition: March 2017 - Volume 64 - Issue 3 - p 472484.

8. Ribeiro, G.F.F. et al. Etiologia e mortalidade por pancreatite aguda: uma revisão sistemática. Arquivos Catarinenses de Medicina, v. 46, n. 4, p. 168-181, 2017.

9. Menin, F.A.; Petreca, A.; Teixeira, R.L. Pancreatite aguda biliar na infância. Rev. Col. Bras. Cir., Rio de Janeiro , v. 33, n. 5, p. 341342, Oct. 2006.

10.Sánchez-Ramírez, C.A.; Larrosa-Haro, A.; Flores-Martínez, S.; SánchezCorona, J.; Villa-Gómez, A.; Macías-Rosales, R. Acute and recurrent pancreatitis in children: etiological factors. Acta Pediatr. 2007;96:534-7

11. Abu-El-Haija, M.; Kumar, S.; Quiros, J.A.; Balakrishnan, K.; Barth, B.; Bitton, S.; Eisses, F.J.; Foglio, J.E.; Fox, V.; Francis, S.; Freeman, J.A.; Gonska, T.; Grover, S.A.; Husain, Z.S.; Kumar, R.; Lapsia, S.; Lin, T.; Liu, Y.Q.; Maqbool, A.; Sellers, M.Z.; Szabo, F.; Uc, A.; Werlin, L.S.; Morinville, D.V. The Management of Acute Pancreatitis in the Pediatric Population: A Clinical Report from the NASPGHAN Pancreas Committee; J Pediatr Gastroenterol Nutr. 2018 Jan; 66(1): 159-176.

Enviado: Junho, 2019.

Aprovado: Setembro, 2019. 